\title{
Equivalence of the adiabatic approximation and the Born approximation for excitations in slowly colliding asymmetric systems
}

\author{
D H Jakubassa and P A Amundsen \\ Institute of Physics, University of Oslo, Oslo, Norway
}

Received 20 August 1979

\begin{abstract}
Within a full quantum-mechanical description we show that the recoil term in the Born approximation can be identified with the perturbation by the target motion in the first-order adiabatic approximation if $Z_{1} / Z_{2} \rightarrow 0$. Moreover, when the excitation is restricted to small internuclear distances, the approximations are equivalent.
\end{abstract}

For the calculation of ionisation or excitation of atoms by heavy particles in slow collisions two different methods based on first-order perturbation theory are commonly in use (Briggs and Taulbjerg 1978). The (distorted-wave) Born approximation is applicable as long as the projectile charge $\left(Z_{1}\right)$ is much smaller than the target charge $\left(Z_{2}\right)$. The adiabatic (Born-Oppenheimer) approximation can be used when the collision velocity $(v)$ is much smaller than typical electronic velocities of the initial state. For a slow, asymmetric collision both methods are applicable and should thus be equivalent. This equivalence has recently been proved in the semiclassical (or impact parameter Born) approximation (Amundsen 1978a), where the nucleus-nucleus interaction is treated classically and independently of the electronic degrees of freedom. The important point in the proof was to include the internuclear recoil term in the Hamiltonian of the Born approximation, as the target atom constitutes a non-inertial frame of reference during the collision. In the present letter we extend the proof to a fully quantum-mechanical treatment of the excitation process. Atomic units $\left(\hbar=m_{\mathrm{e}}=\right.$ $e=1$ ) are used throughout the paper.

The Hamiltonian of the problem can generally be written

$$
H=H_{\mathrm{N}}+H_{\mathrm{e}}+H_{\mathrm{R}}
$$

where $H_{\mathrm{N}}=T_{\mathrm{N}}+V_{\mathrm{N}}$ describes the relative motion of the nuclei corresponding to an internuclear potential $V_{\mathrm{N}}$, and

$$
H_{\mathrm{e}}=T_{\mathrm{e}}+V_{1}(\boldsymbol{r}-\alpha \boldsymbol{R})+V_{2}(\boldsymbol{r}+\beta \boldsymbol{R})
$$

is the electronic part, where $\alpha$ and $\beta=1-\alpha$ defines the choice of origin for the electron coordinate $\boldsymbol{r}$ along the internuclear axis $\boldsymbol{R}$. $H_{\mathrm{R}}$ represents the recoil contribution which has to be included if $\alpha$ and $\beta$ do not correspond to the heavy-particle centre-of-mass frame, $\alpha=\alpha_{0}=M_{2} /\left(M_{1}+M_{2}\right), M_{1}$ and $M_{2}$ being the projectile and target mass, respectively. As in the semiclassical approximation (Amundsen 1978b) the centre of coordinates for the electron can be moved from the centre of mass along $\boldsymbol{R}$ by unitary 
operators (uniquely defined up to a phase):

$$
\begin{aligned}
& U=\exp \left[-\mathrm{i}\left(\alpha-\alpha_{0}\right) \boldsymbol{R} \cdot \boldsymbol{p}\right] \exp \left[\mathrm{i} \mu^{-1}\left(\alpha-\alpha_{0}\right) \boldsymbol{P} \cdot \boldsymbol{r}\right] \\
& \mu^{-1}=M_{1}^{-1}+M_{2}^{-1} \ll 1
\end{aligned}
$$

where $\boldsymbol{P}$ and $\boldsymbol{p}$ are the momenta conjugate to $\boldsymbol{R}$ and $\boldsymbol{r}$, respectively. This transformation of the Hamiltonian leads to the term $H_{\mathrm{R}}$ which is given by

$$
H_{\mathrm{R}}=\mu^{-1}\left(\alpha-\alpha_{0}\right) r \nabla_{R} V_{\mathrm{N}}(\boldsymbol{R})
$$

to the leading order in $\mu^{-1}$. This is identical to the exact semiclassical result by virtue of the equation of motion $\mu \ddot{\boldsymbol{R}}=-\nabla_{R} V_{\mathrm{N}}(\boldsymbol{R})$.

In the Born approximation the unperturbed electronic wavefunctions are chosen to be eigenfunctions $\psi_{n}^{\mathrm{T}}(\boldsymbol{r})$, corresponding to an energy $\epsilon_{n}^{\mathrm{T}}$, of $T_{\mathrm{e}}+V_{2}(\boldsymbol{r})$ centred at the target, so that $\alpha=1$. One then expands the total wavefunction as

$$
\psi=\sum_{n, m} a_{n m}^{\mathrm{T}}(t) \chi_{m}(\boldsymbol{R}) \mathrm{e}^{-\mathrm{i} E_{m} \mathrm{t}} \psi_{n}^{\mathrm{T}}(\boldsymbol{r}) \mathrm{e}^{-\mathrm{i} \epsilon_{n}^{\mathrm{T} t}}
$$

The nuclear wavefunction $\chi_{m}(\boldsymbol{R})$ (energy $E_{m}$ ) is an eigenfunction of $H_{\mathrm{N}}$. Inserting this expansion into the Schrödinger equation one finds the first order transition amplitude from an initial state $\chi_{i} \psi_{i}$ to a final state $\chi_{f} \psi_{f}(f \neq i)$ as

$$
\begin{aligned}
a_{f f}^{T}=-2 \pi \mathrm{i} \delta( & \left.E_{f}-E_{i}+\epsilon_{f}^{\mathrm{T}}-\epsilon_{i}^{\mathrm{T}}\right) \\
& \times\left(\int \mathrm{d} \boldsymbol{R} \chi_{f}^{*} \chi_{i}\left\langle\psi_{f}^{\mathrm{T}}\left|V_{1}(\boldsymbol{r}-\boldsymbol{R})\right| \psi_{i}^{\mathrm{T}}\right\rangle+\frac{\beta_{0}}{\mu}\left\langle\chi_{f}\left|\left(\nabla_{R} V_{\mathrm{N}}\right)\right| \chi_{i}\right\rangle\left\langle\psi_{f}^{\mathrm{T}}|\boldsymbol{r}| \psi_{i}^{\mathrm{T}}\right\rangle\right)
\end{aligned}
$$

where the last term is the recoil term.

In the adiabatic approximation one expands instead the total wavefunction after a set of eigenfunctions $\psi_{n}^{\mathrm{M}}(\boldsymbol{R}, \boldsymbol{r})$ (energy $\epsilon_{n}^{\mathrm{M}}(R)$ ) of $H_{\mathrm{e}}$ centred at the centre of charge of the heavy particles, i.e. $\alpha=Z_{2} /\left(Z_{1}+Z_{2}\right)$. By an expansion similar to equation (5) the first order transition amplitude becomes

$$
\begin{array}{rl}
a_{f f}^{\mathrm{M}}=\frac{2 \pi \mathrm{i}}{\mu} \int \mathrm{d} & \boldsymbol{R}\left\{\delta ( E _ { f } - E _ { i } + \epsilon _ { f } ^ { \mathrm { M } } ( R ) - \epsilon _ { i } ^ { \mathrm { M } } ( R ) ) \left[\chi_{f}^{*}\left(\nabla_{R} \chi_{i}\right)\left\langle\psi_{f}^{\mathrm{M}}\left|\nabla_{R}\right| \psi_{i}^{\mathrm{M}}\right\rangle\right.\right. \\
\left.+\chi_{f}^{*} \chi_{i}\left(\frac{1}{2}\left\langle\psi_{f}^{\mathrm{M}}\left|\nabla_{R}^{2}\right| \psi_{i}^{\mathrm{M}}\right\rangle-\mu\left\langle\psi_{f}^{\mathrm{M}}\left|H_{R}\right| \psi_{i}^{\mathrm{M}}\right\rangle\right)\right] \\
\left.-\delta^{\prime}\left(E_{f}-E_{i}+\epsilon_{f}^{\mathrm{M}}(R)-\epsilon_{i}^{\mathrm{M}}(R)\right)\left(\nabla_{R} \epsilon_{i}^{\mathrm{M}}(R)\right) \chi_{f}^{*} \chi_{i}\left\langle\psi_{f}^{\mathrm{M}}\left|\nabla_{R}\right| \psi_{i}^{\mathrm{M}}\right\rangle\right\} .
\end{array}
$$

The term involving $H_{\mathrm{R}}$ vanishes for collision systems where the centre of mass coincides with the centre of charge.

The first term of equation (7) can be rewritten by using

$$
\begin{aligned}
\left\langle\psi_{f}^{\mathrm{M}}\left|\nabla_{R}\right| \psi_{i}^{\mathrm{M}}\right\rangle= & \frac{1}{\epsilon_{i}^{\mathrm{M}}(R)-\epsilon_{f}^{\mathrm{M}}(R)}\left\langle\psi_{f}^{\mathrm{M}}\left|\left[\nabla_{R}, H_{\mathrm{e}}\right]\right| \psi_{i}^{\mathrm{M}}\right\rangle \\
= & \frac{1}{\epsilon_{i}^{\mathrm{M}}(R)-\epsilon_{f}^{\mathrm{M}}(\boldsymbol{R})}\left(\left\langle\psi_{f}^{\mathrm{M}}\left|\left(\nabla_{R} V_{1}(\boldsymbol{r}-\alpha \boldsymbol{R})\right)\right| \psi_{i}^{\mathrm{M}}\right\rangle\right. \\
& +\left\langle\psi_{f}^{\mathrm{M}}\right|\left(\nabla_{\boldsymbol{R}} V_{2}(\boldsymbol{r}+\beta \boldsymbol{R})\left|\psi_{i}^{\mathrm{M}}\right\rangle\right) .
\end{aligned}
$$

In the asymmetric limit $Z_{1} / Z_{2} \ll 1$, the term involving $V_{2}$, i.e. the term arising from the motion of the target nucleus, can be cast into a form that corresponds to the recoil term in the Born approximation. In this limit we can replace $\psi^{\mathrm{M}}$ by $\psi^{\mathrm{T}}$ and $\epsilon^{\mathrm{M}}(R)$ by $\epsilon^{\mathrm{T}}$, 
because the difference is of the order of $Z_{1} / Z_{2}$. (It is true that these wavefunctions are centred at different origins, but the corresponding translational factors also represent corrections of the order of $Z_{1} / Z_{2}$.) We can thus write

$$
\begin{aligned}
\left\langle\psi_{f}^{\mathrm{M}}\left|\left(\nabla_{R} V_{2}\right)\right| \psi_{i}^{\mathrm{M}}\right\rangle & =\beta\left\langle\psi_{f}^{\mathrm{M}}\left|\left(\nabla_{r} V_{2}\right)\right| \psi_{i}^{\mathrm{M}}\right\rangle \\
& =\beta\left(\boldsymbol{\epsilon}_{f}^{\mathrm{T}}-\boldsymbol{\epsilon}_{i}^{\mathrm{T}}\right)^{2}\left\langle\psi_{f}^{\mathrm{T}}|\boldsymbol{r}| \psi_{i}^{\mathrm{T}}\right\rangle+\mathrm{O}\left(\left(Z_{1} / Z_{2}\right)^{2}\right) .
\end{aligned}
$$

In the last step we have also used that $\psi_{n}^{\mathrm{T}}$ is an eigenfunction of $T_{\mathrm{e}}+V_{2}$ and replaced the acceleration form of the dipole matrix element by the coordinate form. The matrix element in equation (9) is now independent of $\boldsymbol{R}$, so that the $\boldsymbol{R}$ integration in equation (7) can be carried out for this term, yielding the dipole matrix element:

$$
\left\langle\chi_{f} \boldsymbol{\nabla}_{R} \mid \chi_{i}\right\rangle=\frac{1}{E_{i}-E_{f}}\left\langle\chi_{f}\left|\left(\nabla_{R} V_{N}\right)\right| \chi_{i}\right\rangle .
$$

Combining the target term of equation (8) with the term containing $H_{\mathrm{R}}$, we thus arrive at a term identical to the recoil term of equation (6).

As the second and the last term of equation (7) are of the order of $\left(Z_{1} / Z_{2}\right)^{2}$ and thus can be neglected in the asymmetric limit, it remains to prove that the first (projectile) term of equation (8) corresponds to the perturbing potential in equation (6). To do this we note that for an inelastic process the transition amplitude obtains its main contributions from distances of the order of

$$
R \simeq \frac{v}{\Delta E}
$$

where $\Delta E$ is the energy transfer. For the adiabatic approximation to be applicable, $v$ should be small compared with typical electron velocities of the initial state. If $\Delta E$ is of the order of the binding energy of the initial state, equation (11) shows that $R$ is then much smaller than the initial shell radius. We can therefore expand $V_{1}$ in the first term of equation (8) as

$$
\begin{aligned}
\boldsymbol{\nabla}_{R} V_{1}(\boldsymbol{r}-\alpha \boldsymbol{R}) & =-\alpha \nabla_{r} V_{1}(\boldsymbol{r}-\alpha \boldsymbol{R}) \\
& =-\alpha \nabla_{r} V_{1}(\boldsymbol{r})+\mathrm{O}(R) .
\end{aligned}
$$

As the factor $\mu^{-1} \nabla_{R} \chi_{i}$ in equation (7) is proportional to $v$ and thus by equation (11) proportional to $R$, we can neglect terms of the order of $R$ in this expansion. Inserting this into equation (7), the projectile contribution gives rise to the amplitude

$$
\begin{aligned}
-\alpha \frac{2 \pi \mathrm{i}}{\mu} \frac{\delta\left(E_{f}-E_{i}+\boldsymbol{\epsilon}_{f}^{\mathrm{T}}-\boldsymbol{\epsilon}_{i}^{\mathrm{T}}\right)}{\boldsymbol{\epsilon}_{i}^{\mathrm{T}}-\boldsymbol{\epsilon}_{F}^{\mathrm{T}}}\left\langle\chi_{f}\left|\nabla_{\boldsymbol{R}}\right| \chi_{i}\right\rangle\left\langle\psi_{f}^{\mathrm{T}}\left|\left(\nabla_{r} V_{1}(\boldsymbol{r})\right)\right| \psi_{i}^{\mathrm{T}}\right\rangle \\
=\alpha 2 \pi \mathrm{i} \delta\left(E_{f}-E_{i}+\boldsymbol{\epsilon}_{f}^{\mathrm{T}}-\boldsymbol{\epsilon}_{i}^{\mathrm{T}}\right)\left\langle\chi_{f}|\boldsymbol{R}| \chi_{i}\right\rangle\left\langle\psi_{f}^{\mathrm{T}}\left|\left(\boldsymbol{\nabla}_{r} V_{1}(\boldsymbol{r})\right)\right| \psi_{i}^{\mathrm{T}}\right\rangle .
\end{aligned}
$$

Finally we can make a Taylor expansion of $V_{1}(\boldsymbol{r}-\alpha \boldsymbol{R})$ and write

$$
-\alpha \boldsymbol{R} \nabla_{r} V_{1}(\boldsymbol{r})=V_{1}(\boldsymbol{r}-\alpha \boldsymbol{R})-V_{1}(\boldsymbol{r})+\mathrm{O}\left(\boldsymbol{R}^{2}\right) .
$$

Inserting this into equation (13), the term containing $V_{1}(\boldsymbol{r})$ will vanish due to the orthogonality of the nuclear states, and taking $\alpha \rightarrow 1$ we are left with the first term of equation (6). The proof is thus complete.

As a final remark we note that in the derivation of equation (13) together with equation (14) we nowhere needed the fact that $Z_{1} / Z_{2} \ll 1$. As equation (11) is generally valid, we can perform this step for any charge ratio and also include the target term. The 
only modification is that $\psi_{n}^{\mathrm{M}}$ should not be replaced by $\psi_{n}^{\mathrm{T}}$, but instead by wavefunctions corresponding to the united atom limit of $\psi_{n}^{\mathrm{M}}, \psi_{n}^{\mathrm{UA}}$. Also in this small- $R$ limit the second and the last term of equation (7) will vanish, and we are left with the quantal version of Briggs' model (Briggs 1975)

$a_{f f}^{U A}=-2 \pi \mathrm{i} \delta\left(E_{f}-E_{i}+\epsilon_{f}^{\mathrm{UA}}-\epsilon_{i}^{\mathrm{UA}}\right)\left\langle\chi_{f} \psi_{f}^{\mathrm{UA}}\left|V_{1}(\boldsymbol{r}-\alpha \boldsymbol{R})+V_{2}(\boldsymbol{r}+\beta \boldsymbol{R})+H_{\mathrm{R}}\right| \chi_{i} \psi_{i}^{\mathrm{UA}}\right\rangle$.

We would like to thank J S Briggs for a stimulating discussion.

\section{References}

Amundsen P A 1978a PhD thesis University of Bergen 1978b J. Phys. B: Atom. Molec. Phys. $113197-220$

Briggs J S 1975 J. Phys. B: Atom. Molec. Phys. 8 L485-8

Briggs J S and Taulbjerg K 1978 Tópics in Current Physics vol 5, ed I A Sellin (Berlin: Springer) pp 105-53 